\title{
Linx
}

Revue des linguistes de l'université Paris X Nanterre

$44 \mid 2001$

Spécificité et histoire des discours sémiotiques

\section{La sémiologie saussurienne, entre le CLG et la recherche sur la légende}

\section{Michel Arrivé}

\section{(2) OpenEdition}

\section{Journals}

Édition électronique

URL : http://journals.openedition.org/linx/1015

DOI : 10.4000/linx.1015

ISSN : 2118-9692

\section{Éditeur}

Presses universitaires de Paris Nanterre

\section{Édition imprimée}

Date de publication : 1 juin 2001

Pagination : 13-27

ISSN : 0246-8743

\section{Référence électronique}

Michel Arrivé, "La sémiologie saussurienne, entre le CLG et la recherche sur la légende », Linx [En ligne], 44 | 2001, mis en ligne le 05 juillet 2012, consulté le 30 avril 2019. URL : http:// journals.openedition.org/linx/1015; DOI : 10.4000/linx.1015

Ce document a été généré automatiquement le 30 avril 2019.

Département de Sciences du langage, Université Paris Ouest 


\title{
La sémiologie saussurienne, entre le CLG et la recherche sur la légende
}

\author{
Michel Arrivé
}

1 Mon intention n'est pas de contribuer à l'histoire de la sémiologie ni de la sémiotique. J'en serais d'ailleurs empêché par la spécificité de mon sujet. Et même doublement empêché. Pour le CLG, son rôle fondateur dans l'histoire des deux disciplines jumelles est dès maintenant pour l'essentiel bien décrit. Après que Barthes et Greimas se sont expliqués, plus ou moins abondamment, sur ce qu'ils doivent au CLG, de nombreux travaux ont fait le point (voir, en dernier lieu, Arrivé 2000a et b). Pour la recherche sur la légende - désormais $L E G$, par référence à l'édition de Marinetti \& Meli 1986 - les faits sont entièrement différents. D'une part parce que l'enseignement proprement sémiologique est, dans cette recherche, non achevé, réduit à quelques dizaines de pages dispersées et presque toujours restées à l'état d'ébauche. Il faut les exhumer de très longues dissertations d'histoire événementielle ou de spéculations onomastiques. Non, certes, que ces éléments soient privés de relations avec le projet sémiologique : mais ces relations - sur lesquelles je reviendrai - sont difficiles à apercevoir d'emblée. En outre, il faut rappeler que si le CLG est accessible, sous sa forme dite «standard» depuis 1916, la recherche sur la légende n'a été très progressivement dévoilée qu'à partir de 1957. Il a fallu attendre 1986 pour en lire l'édition encore incomplète, philologiquement imparfaite, et de toute façon restée très confidentielle que je viens de signaler. Le caractère même du travail et le retard avec lequel il a été parcimonieusement révélé expliquent que son influence sur la fondation et l'évolution de la sémiotique soit restée marginale. Pour ne citer que les deux noms allégués plus haut, je crois pouvoir avancer que ni Barthes ni Greimas - qui connaissaient l'existence de la recherche - n'en ont tenu compte de façon significative dans leurs travaux.

2 Ainsi, je ne ferai pas d'histoire. Que vais-je donc faire? Un travail à la fois nécessaire, difficile et ambitieux : en prenant pour point de départ l'exemple de Saussure, réfléchir sur le problème des relations entre la linguistique et la sémiologie. Je ne suis certes pas le premier, spécifiquement à propos de Saussure. Tout récemment, le livre de Claudine Normand (2000) et celui de Johannes Fehr (2000), pour qui le problème est central, sont 
revenus sur une question déjà abordée par de nombreux autres chercheurs, au premier rang desquels d'Arco Silvio Avalle (1973), Rudolf Engler (1974-1975 et 1980) et Kim (1993). Francis Gandon (2002) se prépare aussi à révéler ses positions sur le problème. Si j'interviens après eux, c'est que j'estime que tout n'a pas été dit, tant sur le problème de l'origine que sur la notion fascinante et problématique d'« être inexistant» : car, on le verra, c'est le statut paradoxal qui, dans la recherche, est affecté par Saussure au « SIGNE, au sens philosophique ». renvoie à l'illustre passage des pages 33 à 35 , où est mise en place la sémiologie et où est posé, de façon particulièrement ardue - «on tourne dans un cercle», est-il dit p. 34 : promenade familière à Saussure - le problème de ses relations avec la linguistique. La seconde entrée de l'index renvoie à la page 100 , où se trouve posé le problème de l'appartenance à la sémiologie des systèmes de "signes entièrement naturels " - expression saussuriennement oxymorique qui n'apparaît que dans le texte de la vulgate et non dans les sources manuscrites. Le texte indique que cette appartenance ne peut être que marginale. Aussitôt après est prononcé un verdict qui est assez fortement modulé dans les sources manuscrites:

La linguistique peut devenir le patron général de toute sémiologie, bien que la langue ne soit qu'un système particulier (CLG:101).

En ce point, les sources insistent sur le caractère aléatoire du choix de la linguistique comme «patron général ». Toutefois aucun autre système de signes n'est allégué comme candidat de rechange à la fonction de « patron général ».

la sémiologie, souvent sous les espèces de l'adjectif sémiologique, apparaît en plusieurs points du CLG non signalés par l'index. Ainsi p. 111 se pose, à propos de l'esperanto ${ }^{1}$ et de ses éventuelles mutations, le problème du signe dans le temps : le signe, on s'en souvient, est à la fois affecté, selon le titre du chapitre de l'édition standard, par l'« immutabilité et la mutabilité ». Ce problème, comme on verra, sera central dans la construction des relations entre linguistique et sémiologie :

La continuité du signe dans le temps, liée à l'altération dans le temps, est un principe de la sémiologie générale (Les sources concordent à peu près exactement avec le texte standard).

7 À la page 149, est posé un problème fondamental que nous ne ferons guère qu'effleurer aujourd'hui : celui de la différence éventuelle de statut entre les unités de la langue et les unités des autres systèmes sémiologiques. J'ai abordé ce problème capital dans Arrivé 1998.

8 Les sources manuscrites, qui, comme on sait, comportent aussi des réflexions extérieures aux trois Cours de 1907 à 1911, donnent à la sémiologie une place plus importante². Je signale quelques-uns de ces fragments avant de citer celui qui me paraît le plus 
important : Saussure 1989 : I-147 (N 12, n 1086) ; I-148-149 (N 12, $\left.\mathrm{n}^{\circ} 1088-1091\right)$; I-273 (N $\left.12, \mathrm{n}^{\circ} 1950\right) ; 1990$ : II-47. Le segment le plus pertinent est sans doute celui-ci :

La nature du signe ne peut donc se voir que dans la langue, et cette nature se compose des choses qu'on étudie le moins.

C'est pour cela qu'on ne voit pas à première vue la nécessité ou l'utilité particulière d'une science sémiologique, quand il est question de la langue à des points de vue généraux, philosophiques; quand on étudie autre chose avec $^{3}$ la langue (Saussure 1989 I-51 (II R 22, n 313-314); voir CLG : 34, où cette position est rejetée).

On aperçoit d'emblée la contradiction qui s'installe entre la position énoncée plus haut et celle qui se met en place ici : la linguistique est donnée dans le premier segment comme le patron de toute sémiologie possible. Dans le second, le signe linguistique est présenté comme absolument spécifique, en sorte qu'une éventuelle sémiologie ne peut être que non pertinente à son égard. Nous retrouverons cette contradiction - et serons peut-être en mesure de l'expliquer - quand nous ferons intervenir les unités sémiologiques de la légende.

Mais la sémiologie apparaît dans les préoccupations linguistiques de Saussure bien avant les trois Cours de Genève. Engler (1980: 4), puis Fehr (2000: 110 n.4) remarquent que c'est en 1894, dans le projet d'article relatif à Whitney, qu'apparaît pour la première fois le mot sémiologie. Il a dans ce texte la particularité d'être pris non avec le sens de «science des signes », mais avec celui de « langage-objet » : quand il observe « la si complexe nature de la sémiologie particulière dite langage » (Saussure 1989 : I-197), Saussure pose - déjà - la spécificité du langage parmi les autres objets possibles de la sémiologie. On sait d'autre part que Saussure a présenté la sémiologie - cette fois avec le sens de «science des signes " - de façon assez explicite et assez convaincante à son collègue Adrien Naville pour que celui-ci lui donne une place centrale, en 1901, dans sa Nouvelle classification des sciences (1901-1991: 104) ${ }^{4}$.

11 Quant à l'intérêt de Saussure pour la légende - et pour certains discours de type comparable, par exemple la mythologie - il est également fort ancien. A. Cuny rapporte - il est vrai en 1937, soit près de soixante ans après - que le très jeune Saussure, affecté par l'échec, en Allemagne, de son Mémoire, fut tenté, dès 1880, de se tourner vers l'étude de l'épopée germanique. Moins loin dans le passé, en 1894, on trouve, inséré au sein du projet d'article sur Whitney, un développement à proprement parler fascinant sur les noms des dieux de la mythologie indienne et grecque (Saussure 1990 : II-25). D'une façon qui annonce les spéculations onomastiques de la recherche sur la légende, Saussure envisage la séparation du nom des Dieux d'avec tout «objet sensible»: inversant la formule traditionnelle numen, nomen - «la divinité, c'est son nom » - Saussure avance que

du sort du nomen dépend très décisivement, et pour ainsi dire de seconde en seconde celui du numen.

Pour Saussure, le nom, c'est la divinité, telle qu'elle se trouve insérée dans le système de signes que constitue aussi la mythologie. Et les changements de son nom impliqueront pour elle des mutations proportionnelles. Au risque de paraître excessivement vétilleux, j'insiste sur l'étonnante précision temporelle « de seconde en seconde » : l'évolution dans le temps du nomen - c'est-à-dire du numen - est à proprement parler fulgurante. De quel temps est-il donc question ici ? De celui qui modifie au cours de l'histoire les noms des divinités? Mais il ne se mesure pas en " secondes ». Ne serait-ce pas plutôt celui qui, dans le discours, sépare, éventuellement d'une «seconde" à l'autre, les occurrences successives d'un même nom? Mais a-t-il pour effet de modifier les noms? La solution est 
sans doute de poser que ces deux manifestations du temps, apparemment différentes, n'en font réellement qu'une. On retrouve ici le problème illustré dans le CLG par une comparaison qui passe souvent inaperçue, tant elle paraît paradoxale : après avoir posé la différence entre les emplois successifs du Messieurs d'un conférencier - séparés l'un de l'autre par quelques «secondes" - Saussure la compare à celle qui s'observe entre le latin calidum et le français chaud - séparés par une vingtaine de siècles: «le second problème n'est en effet qu'un prolongement et une complication du premier » (CLG:250) J'ajoute qu'on retrouvera ce problème du temps dans quelques instants, à propos de ces deux autres types de signes que sont la personne mythique et la lettre de l'alphabet.

L'année 1904 marque un moment important dans la réflexion de Saussure sur la légende. Le 15 décembre, il prononce, devant les membres de la Société d'histoire et d'archéologie de Genève, une conférence sur «les Burgondes et la langue burgonde en pays roman ». L'examen de certains toponymes vaudois, d'origine apparemment burgonde, lui permet d'avancer une hypothèse hardie :

[Si l'origine burgonde de ces toponymes était validée], on aurait à se demander quelle part l'Helvétie burgonde peut avoir eue dans la genèse et la propagation de la légende épique des Nibelungen (1921-1984: 606).

On le voit: dans cette unique trace publiée de son vivant de sa réflexion sur la légende germanique, Saussure en envisage l'origine référentielle : les événements rapportés ont eu originellement à désigner des événements réels, dans un pays réel, même si les données toponymiques ne permettent pas de situer avec certitude ce pays. On verra plus loin les perplexités - théoriques, et non pas historiques - dans lesquelles cette hypothèse plonge Saussure, et la solution radicale qu'il donne à cette difficulté.

C'est sans doute à partir de cette même année 1904 - pendant laquelle il donne un cours public sur le Nibelungenlied - que Saussure commence à rédiger les innombrables feuillets qu'il consacre à sa recherche : pas moins de 820, selon le dénombrement de Fehr (2000: 247). Même si, comme on vient de l'entrevoir, les enquêtes historiques et les manipulations onomastiques y sont envahissantes, la sémiologie est très fréquemment alléguée, comme on le verra dans les citations que je ferai dans la suite.

Concluons sur ces détails chronologiques: la recherche sémiologique sur la légende est pour l'essentiel coextensive dans le temps avec la recherche linguistique.

Qu'en est-il maintenant de la relation entre les deux recherches contemporaines telles qu'elles se manifestent dans les deux corpus? On peut le dire d'un mot: elle est totalement asymétrique. On constate en effet que, sauf erreur ou oubli, le travail sur la légende n'est jamais allégué quand, dans le CLG, il est question de la sémiologie. Saussure consent parfois à donner des exemples de «systèmes de signes » autres que la langue : il les choisit alors dans les deux classes suivantes :

18 a) D'une part des systèmes dérivés de la langue, ou en tout cas envisagés comme tels dans l'une des deux conceptions que s'en fait Saussure. Ce sont l'écriture et l'alphabet des sourds-muets. On sait - et on verra, fugitivement, plus bas - que cette conception n'est pas la seule dans la réflexion saussurienne.

19 b) D'autre part des systèmes régionaux tels que les rites symboliques, les formes de politesse, les signaux militaires. À la seule réserve des derniers - sur la nature exacte desquels il n'est pas aisé de se prononcer: pavillons de marins? ou sonneries de trompettes? - il s'agit de systèmes de signes au moins partiellement motivés : on a vu plus haut que leur appartenance à la sémiologie est mise en cause. 
20 Quoi qu'il en soit, on ne peut que remarquer la pauvreté de cette exemplification. Et s'étonner qu'au moment même où il essaie de mettre en place l'analyse sémiologique de la légende, Saussure ne fasse pas apparaître la légende - non plus que la mythologie dans l'inventaire des « systèmes de signes ».

21 L'étonnement s'accroît encore quand on jette un coup d'œil du côté de la légende. On constate en effet qu'à l'opposé du silence observé dans le CLG à l'égard de la légende,la langue est très fréquemment alléguée dans les réflexions relatives à la légende. Ainsi, la langue est à différentes reprises explicitement donnée comme un objet de la sémiologie, en raison de sa " parenté $»^{6}$ avec la légende :

Ces symboles ${ }^{7}$ [qui composent la légende] sont soumis aux mêmes vicissitudes et aux mêmes lois que toutes les autres séries de symboles, par exemple les symboles qui sont les mots de la langue. Ils font tous partie de la sémiologie (LEG : 30 ; voir aussi 191-192 et 307-308).

Comment s'explique cette dissymétrie entre les deux recherches? Comment se fait-il que d'un côté la langue soit donnée au même titre que la légende comme objet de la sémiologie, alors que de l'autre côté la légende n'est même pas nommée ? La question a l'air d'être futile. J'ai la faiblesse de penser qu'elle ne l'est pas : elle va nous permettre de repérer à la fois ce qui rapproche les deux objets et ce qui les oppose.

Revenons un instant à la toponymie telle que Saussure la présente dans sa communication de décembre 1904. Les noms de lieux burgondes du canton de Vaud et des régions voisines suggèrent pour le Nibelungenlied une origine géographique et événementielle. Cette suggestion fournie par la toponymie est reprise à titre d'hypothèse de travail en plusieurs points de la recherche, et notamment au moment où - sans doute dans l'intention de donner à son travail la forme d'un livre - Saussure pense à un titre. Ce titre est au plus haut point explicite : Histoire et légende. Étude sur l'origine des traditions germaniques connues sous le nom de Heldensage (LEG : 183). Et le programme qui est assigné par ce titre est résumé de façon non moins explicite :

Le titre de ce volume indique que nous supposons un lien historique entre les événements qui se sont déroulés de 443 à 534 dans le royaume fondé en Savoie par les Burgondes, et connu sous le nom de ${ }^{\text {er }}$ Royaume de Burgondie. Tel [sic] est en effet notre idée et notre conviction.

Ce n'est pas le Gundacharius mort en 434, mais le Gundobadus mort en 516 qui sera pour nous le Gunther central, expliquant l'épopée burgonde (LEG : 130).

On ne saurait être plus clair, notamment à propos du personnage légendaire Gunther : il est - le sera de Saussure n'est nullement une atténuation de prudence- le personnage historique qui porta effectivement le nom de Gundobadus.

Cette définition du " personnage » par son référent originel n'a rien de spécifiquement original dans la recherche de l'époque sur la légende. Mais elle fait gravement problème dans le cadre de la sémiologie saussurienne. Car, on vient de l'apercevoir par une précédente citation, le caractère sémiologique de la légende lui vient de ce que les « unités » qu'elle comporte, les « personnages », sont, au même titre que « les mots de la langue ", des « symboles ", c'est-à-dire des « signes » si on tient compte de la mutation de la terminologie saussurienne. Au delà de ce problème de terminologie, on voit la difficulté : les «mots de la langue » - autrement dit les « signes » - sont définis non pas par la chose à laquelle le hasard des faits de parole peut les attacher provisoirement, mais par la relation entre les deux faces que sont, dans l'une des nombreuses terminologies successives de Saussure, le «concept» et l'«image acoustique». Rien de tel pour le 
personnage de la légende : il est « expliqué » par la relation originelle de son nom avec le personnage historique qu'il a originellement désigné. On entre avec cet objet sémiologique d'un type particulier dans le régime adamique de la nomination. Régime qui, on le sait bien, est récusé avec une virulence plus ou moins intense en différents points de la réflexion de Saussure. Ainsi dans l'une des «Notes item» le voit-on considérer avec mépris « ce qu'il y a de plus grossier dans la sémiologie : le cas où elle est, par le hasard des objets désignés, une simple onymique » - c'est-à-dire une relation entre une chose et un nom (Saussure, 1990: II-36). On remarque toutefois que le mépris de l'auteur pour ces "cas grossiers" n'entraîne pas pour lui leur exclusion de la "sémiologie", mais simplement leur isolement dans une zone marginale de celle-ci : l'« onymique ».

Saussure est encore plus explicite dans le passage des sources manuscrites où il allègue explicitement, naturellement pour récuser son geste, la figure de "notre premier père Adam appelant près de lui les divers animaux et leur donnant à chacun leur nom " (Saussure 1989: I-147; l'édition standard a supprimé toute allusion à «notre père Adam »).

En somme, l'unité sémiologique spécifique de la légende présente à titre de double particularité deux caractères indissociables : on peut lui assigner une origine, et cette origine est référentielle. Par là elle s'écarte totalement du statut du signe linguistique. Ce dernier en effet, même s'il a une origine, est d'une nature telle que le problème de cette origine n'a pas à être posé. C'est, entre plusieurs autres, l'illustre passage de la page 105 du CLG et ses étymons, plus explicites encore, illustrés qu'ils sont par la comparaison avec la source du Rhône, dans les Sources manuscrites :

La question de l'origine des langues n'a pas l'importance qu'on lui donne. Cette question n'existe même pas. Question de la source du Rhône : puérile! Le moment de la genèse n'est lui même pas saisissable : on ne le voit pas (Saussure 1989 : I-160).

Je remarque au passage - en vous demandant de la mettre en mémoire pour la suite - la spécificité de la position de Saussure sur ce problème de l'origine. En effet, il ne dénie pas aux langues une origine : il lui arrive même d'évoquer, fugitivement, il est vrai, l'homme «sans langage articulé » (Saussure 1990: II-16) ou "sans le langage» (II-4), ou plus explicitement encore de songer au "premier jour où une société humaine a parlé» (II-10). Mais ce moment mythique n'a pas lieu d'être pris en compte : pour la langue, le problème de l'origine se confond avec celui de la transmission :

Le moment où l'on s'accorde sur les signes n'existe pas réellement, n'est qu'idéal. Et existerait-il qu'il n'entre pas en considération à côté de la vie régulière de la langue (Saussure 1989 : I-160).

On l'a compris : tel qu'il est pour l'instant mis en place, le symbole de la légende n'a pas les caractères du signe linguistique. Ainsi s'explique sans doute, du point de vue philologique, le silence observé dans le CLG sur la sémiologie légendaire : dans l'état que nous venons d'observer, elle est totalement déviante par rapport à la linguistique.

Ce silence n'est rien d'autre que l'indice textuel d'une difficulté théorique majeure. Elle tient dans la coexistence de deux points de vue opposés à l'égard des relations entre les signes linguistiques et ceux des autres systèmes, notamment ceux de la légende.

D'un côté le signe linguistique est donné comme un exemple parmi d'autres, de même nature que lui. C'est le point de vue adopté notamment dans l'illustre passage de la page 33 , où se trouve posée la parenté entre la langue et ces autres systèmes de signes que sont par exemple l'écriture et les « signaux militaires ». 
de la langue - est présenté comme un objet absolument spécifique :

Le langage est un objet situé en dehors de toute comparaison et non classé dans

l'esprit des linguistes ni dans l'esprit des philosophes (Saussure 1990 : II-41).

... ou, de façon plus explicite encore :

Il n'existe pas d'objet tout à fait comparable à la langue qui est un être très complexe, et c'est ce qui fait que toutes les comparaisons et toutes les images aboutissent régulièrement à nous en donner une idée fausse par quelques point (Saussure 1990 : II-6).

De cette duplicité des points de vue à l'égard du signe s'ensuit une divergence, déjà entrevue plus haut à propos de la p. $34 \mathrm{du} C L G$, sur le problème des relations entre linguistique et sémiologie. On ne s'étonne pas d'observer ici encore un phénomène de dissymétrie. La pertinence de la sémiologie à l'égard de la langue est mise en cause en plusieurs points : nous en avons rencontré un plus haut. L'optimisme provisoire - et très modéré - qui semblait régner dans le CLG est abandonné. Mais inversement la pertinence de la linguistique à l'égard des autres systèmes de signes ne semble à aucun moment être sérieusement contestée. La sémiologie reste inefficace à l'égard du signe linguistique, mais la linguistique conserve sa pertinence à l'égard des autres systèmes de signes.

n'avons pas tout à fait fini d'errer à la suite de Saussure dans le cercle infernal des relations entre linguistique et sémiologie. Après avoir expliqué le silence du CLG à l'égard de la légende, il faut maintenant rendre compte de la présence de la langue dans la recherche sur la sémiologie légendaire. On s'en est en effet aperçu: cette présence est explicite et répétitive. Elle fait gravement problème: comment est-il possible de comparer, voire d'assimiler « les symboles de la légende » aux « mots de la langue » - on aura reconnu les termes utilisés dans le fragment de $L E G$ cité plus haut - s'ils sont à ce point différents d'eux ? La réponse est à la fois simple et paradoxale : Saussure a en réserve une autre conception du personnage, symbole de la légende, qui fait effectivement de lui un double du signe linguistique. Si on se souvient de la première conception du symbole, on devine que cette seconde approche du personnage consiste à le séparer, d'un même geste, de son origine et de son référent.

Comment cette nouvelle conception est-elle mise en place? Voyons-le à l'aide d'un exemple. On se souvient que Gunther, dans le texte cité plus haut, est donné avec "conviction" comme défini par son identité avec le personnage historique nommé Gundobadus. Prenons maintenant un autre personnage de la légende, par exemple Hugdietrich, alias Wolfdietrich - la duplicité du nom n'est pas indifférente. Est-il au même titre que Gunther défini par son assimilation avec un personnage historique, pour lui le très réel Théodéric? Point du tout. Saussure va même jusqu'à se gausser cruellement de celui des exégètes - un certain Symons - qui se livre à une telle spéculation. Il le cite et le commente en ces termes :

Que Wolf[Hug]dietrich soit le Théodéric fils de Clovis est incontesté et incontestable ... Symons.

Cette phrase a de quoi rendre rêveur d'abord en dehors de tout fait, parce qu'on ne sait pas, à un point de vue méthodologique, ce qu'elle peut signifier dans le domaine des études mythiques (LEG 191).

J'interromps un instant la citation pour donner à ceux qui le souhaitent le loisir de hurler à la contradiction. Et pour me donner celui de défendre Saussure. Non, il n'y a pas contradiction. Je m'explique. Je ne suis pas de ceux - il y en a - qui récusent totalement l'existence de contradictions dans la pensée de Saussure. Il y a des contradictions chez 
Saussure : elles signalent un certain nombre de nœuds gordiens de sa réflexion, et, peutêtre, de toute réflexion linguistique et/ou sémiotique. Mais ici il n'y en a pas. Ce n'est pas l'assimilation de (Wolf)Hugdietrich à Théodéric qui est mise en cause. Elle est même peutêtre exacte : en tout cas, Saussure ne prend même pas la peine de dire si elle est fausse. Certains passages de la recherche semblent même indiquer qu'il la juge exacte. C'est qu'en réalité, exacte ou fausse, elle est rigoureusement dépourvue de pertinence à l'égard du véritable statut sémiologique de ce "symbole» qu'est le personnage de Wolf (Hug)dietrich, car il faut lui donner ses deux noms. Quel est ce statut? Il convient ici de reprendre le texte de Saussure au point où je l'ai interrompu :

Il est vrai qu'en allant au fond des choses, on s'aperçoit, dans ce domaine, comme dans le domaine parent de la linguistique, que toutes les incongruités de la pensée proviennent d'une insuffisante réflexion sur ce qu'est l'identité lorsqu'il s'agit d'un être inexistant comme le mot, ou la personne mythique, ou une lettre de l'alphabet qui ne sont que différentes formes du SIGNE au sens philosophique (LEG : 191).

On se trouve ici aux prises avec la notion fascinante - et, il faut bien l'avouer, apparemment autocontradictoire - $d^{\prime}$ ' être inexistant $»^{8}$. Comment faut-il l'entendre ? Et comment s'applique-t-elle à ces trois formes «différentes » de "signes » que sont le mot, la personne mythique et - de retour dans l'inventaire des signes - la lettre de l'alphabet ? C'est cette dernière qui est prise comme tertium comparationis entre la langue et la légende. Et c'est cette comparaison qui permet d'approcher la notion litigieuse d'« être inexistant » :

Une lettre de l'alphabet, par exemple une lettre de l'alphabet runique germanique, ne possède par évidence, dès le commencement, aucune autre identité que celle qui résulte de l'association :

a. d'une certaine valeur phonétique,

b. d'une certaine forme graphique,

c. par le nom et les surnoms qui peuvent lui être donnés,

d. par sa place (son numéro) dans l'alphabet.

Si deux ou trois de ces éléments changent, comme cela se produit à tout moment, et d'autant plus rapidement que souvent un changement entraîne l'autre, on ne sait plus littéralement et matériellement ce qui est entendu, ou plutôt [...] (LEG:191).

Deux mots, d'abord, pour rendre compte du retour de l'écriture et spécifiquement de l'alphabet runique dans l'inventaire des objets de la sémiologie. L'écriture est ici conçue selon le modèle qui fait d'elle non un auxiliaire de la langue, mais un système de signes de plein exercice. Dans le CLG, c'est ce système qui est employé, p. 165, pour illustrer, par l'analyse de la lettre $\mathrm{T}$ et de ses différentes variantes, le concept de valeur, qui affecte aussi la langue. C'est de la même façon que l'écriture est ici utilisée, pour donner un exemple concret de données qui, touchant des traits moins apparents, affectent aussi la sémiologie légendaire. Quant au choix de l'alphabet runique, il est surdéterminé. D’une part c'est une écriture germanique effectivement utilisée pour certaines versions scandinaves du Nibelungenlied'. Et d'autre part l'alphabet runique a été sujet dans son histoire à de fréquentes mutations, qui ont effectivement porté sur le nombre des lettres (24, puis 16, puis 23), nécessairement sur leur ordre, sur leurs noms et sur leurs formes ${ }^{10}$. Ces mutations ont été relativement rapides : pas plus de trois siècles et demi, selon Marcel Cohen (1958: 197), pour l'ensemble des modifications alléguées.

On le voit d'après l'analyse de Saussure: le signe qu'est la lettre n'a pas d'existence substantielle. C'est en cela qu'il est qualifié d'« être inexistant ». Car, contrairement à ce qu'avance Avalle (1973 : 43), cela ne l'empêche pas d'exister. Mais il n'accède à son statut que dans la mesure où il « associe » un certain nombre de traits. Encore cette association 
est-elle à tout moment menacée de destruction. Mais à tout moment elle se reconstitue, par la modification des traits qu'elle réunit. Il suffit par exemple que la lettre change de nom pour qu'elle perde son identité et en prenne une autre. Une lettre n'est jamais identique à elle-même. Il en va de même pour cet autre signe - ou symbole: on se souvient qu'ici les deux termes sont équivalents - qu'est le personnage de la légende, lui aussi constitué par l'association à tout instant variable de quelques traits :

[...] chacun des personnages de la légende est un symbole dont on peut faire varier - exactement comme pour la rune - a) le nom, b) la position vis-à-vis des autres, c) le caractère, d) la fonction, les actes. Si un nom est transposé, il peut s'ensuivre qu'une partie des actes sont transposés et réciproquement, ou que le drame tout entier change par un accident de ce genre ( $L E G: 31$ ).

Cet inventaire des "éléments » varie légèrement au cours de la recherche. Saussure y ajoute parfois le «blason» (LEG:194), voire le «casque " (LEG:195). Le nom - à la différence de ce qui se passe pour la lettre - est, sauf erreur, toujours cité en premier. C'est qu'il a pour le personnage légendaire un statut spécifique :

Il n'est pas comme chez un individu vivant une étiquette sur la personne, mais au même rang que les autres choses, et à ce point de vue plus important ; seulement ce qui compense c'est que tandis que les autres caractères de l'individu sont inséparables de lui [...], tout trait de l'être légendaire peut se dissiper au premier souffle avec autant de facilité que son nom ( $L E G: 142)$.

41 Ainsi les signes que sont les personnages de la légende - et, dans des conditions légèrement différentes, les lettres de l'alphabet - n'ont jamais aucune consistance matérielle. Leur être est par essence fugitif et instable. "Fantômes ", "bulles de savon ", même pas: car la bulle de savon "possède du moins son unité physique et mathématique » (LEG: 192). Le signe ne consiste en rien. Il ne tient qu'à la rencontre provisoire et accidentelle de quelques traits voués à tout instant à se désunir. Mais pour constituer sans délai un autre signe.

Encore faut-il prendre une précaution. Le "Temps », qui est indispensable à ces transformations du signe - comment concevoir une transformation hors du temps? n'en est pas la cause :

Comme on le voit au fond l'incapacité à maintenir une identité certaine ne doit pas être mise sur le compte des effets du Temps - c'est là l'erreur remarquable de ceux qui s'occupent des signes, mais est déposée d'avance dans l'être que l'on choye [sic] et observe comme un organisme, alors qu'il n'est que la combinaison fuyante de 2 ou 3 idées ( $L E G:$ 192).

Et c'est cet «être inexistant», cette «bulle de savon », ce "fantôme » qui est, nouveau paradoxe, objet d'amour. Je ne crois pas pousser trop loin la pensée de Saussure en employant ce mot, qu'il n'utilise pas. Il se contente de verbes: tels «choyer", aperçu dans le segment précédent, ou même « chérir »:

L'association - que nous chérissons parfois - n'est qu'une bulle de savon (LEG :

192).

Nous n'en avons pas encore fini avec les paradoxes relatifs au signe de la légende: cet être à la fois « inexistant » et " chéri » en vient parfois à accéder à une sorte de vie, voire de conscience et même de réflexion. C'est ce qui est manifesté en plusieurs points par certains détails d'expression, dans des phrases il est vrai négatives : ainsi le symbole ne "se doute pas» de son appartenance à la sémiologie ( $L E G: 30)$, ou bien "n'a pas un moyen de prouver qu'il est resté le même» (LEG: 192). Qu'en est-il de ces bizarres phénomènes de personnification du symbole dans l'écriture de Saussure? Ne seraient-ils 
pas la marque d'un désir de substance, voire de substance pensante, pour cet «être inexistant » ? Je laisse prudemment la question pendante...

Qu'en est-il maintenant du troisième type de signe, j'entends celui de la langue ? Dans la recherche, il n'est, si j'ai bien lu, qu'allégué, sans être analysé. Pour apercevoir son statut, il convient de confronter deux textes sans doute fort éloignés dans le temps. Le premier, extrait de la recherche sur la $L E G$, envisage avec un optimisme mesuré - on sait que Saussure est rarement porté à l'euphorie - la tâche de décrire l'ensemble des mutations susceptibles d'affecter le signe de la légende :

Donc en principe, on devrait purement renoncer à suivre, vu que la somme des modifications n'est pas calculable (LEG:31).

En 1894, dans le projet d'article relatif à Whitney, Saussure faisait un pronostic littéralement identique à propos du langage :

Ce qui a échappé ici aux philosophes et aux logiciens, c'est que du moment qu'un système de symboles est indépendant des objets désignés, il était sujet à subir, pour sa part, par le fait du temps, des déplacement non calculables pour le logicien (Saussure 1990 : II-23).

Ainsi sont données comme « incalculables » les modifications des signes linguistiques et des signes légendaires. C'est qu'ils sont l'un et l'autre de la même étoffe. Aussi ne s'étonne-t-on pas de voir que les signes de la langue sont qualifiés non pas d'« êtres inexistants », mais - à mon sens, c'est équivalent - de «termes en soi nuls » : c'est dans l'une des « Notes item » que se trouve cette qualification, dans des conditions à vrai dire très surprenantes :

Item. Il y a défaut d'analogie entre la langue et toute autre chose humaine pour deux raisons. 1. La nullité interne des signes. 2. La faculté de notre esprit de s'attacher à un terme en soi nul. (Saussure 1990 : II-38).

Et Saussure poursuit, en un remords à la fois pathétique, parenthétique et énigmatique :

(Mais ce n'était pas ce que je voulais dire d'abord. J'ai dévié.) (Ibid.)

Ce texte, il faut l'avouer, est littéralement labyrinthique. Saussure affecte au signe linguistique un statut identique à celui du symbole de la légende : car on voit mal quelle différence pourrait s'établir entre les expressions "être inexistant» pour la légende et «terme en soi nul» pour le signe linguistique. D'autant qu'il prend soin de noter l'« attachement " paradoxal que l'esprit porte à ce dernier : c'est l' exact équivalent de l'amour porté au symbole légendaire. Et c'est à ce moment même qu'il pose l'unicité absolue de la langue, perdant de vue complètement la légende - comme d'ailleurs l'écriture. Faut-il essayer de se raccrocher au remords de la parenthèse, et spéculer sur ce que Saussure avait l'intention d'écrire avant, comme il dit, de « dévier»? La spéculation est sans doute un peu litigieuse. Il vaut mieux envisager une autre solution. Et essayer de «catalyser » la réflexion saussurienne, en ce point restée muette, ou à tout le moins indirecte. Je m'y aventure. On a vu tout à l'heure que Saussure donnait comme «non calculables» les modifications dans le temps du symbole légendaire comme du signe linguistique. Non calculables? Certes. Mais pas tout à fait au même degré. Pour le symbole légendaire, Saussure, dans un ultime sursaut d'optimisme épistémologique, finit par admettre que tout compte fait, " on peut relativement espérer suivre, même à des grands intervalles de temps et de distance » (LEG:31). Pour le signe linguistique, rien de tel : l'incalculabilité des « déplacements » reste absolue. Pourquoi cette différence ? Tientelle au nombre des éléments dont la réunion fortuite et provisoire constitue à chaque fois le symbole comme le signe? Non: car ces «éléments » sont en nombre à peu près 
équivalent, et fort peu élevé ${ }^{11}$. La différence ne peut guère tenir qu'à un trait fort discrètement allégué par Saussure, et pour l'un seulement des deux objets comparés : le nombre même des signes. Car pour la légende - comme, dans des conditions différentes, pour l'alphabet - il est limité : une vingtaine de signes pour l'alphabet, un peu plus pour la légende si on tient compte d'un cycle de textes. On n'atteint sans doute pas la centaine. Mais pour la langue le nombre de signes est illimité. Surtout chacun de ces signes donne lieu chaque jour, sans interruption, à des milliers d'emplois. Je cite une dernière fois Saussure :

Il faut ajouter que cette chose [la langue] ne peut pas s'interrompre, même dans l'espace de 24 heures, et chaque élément en est réédité des milliers de fois en ce temps (Saussure $1990:$ II-21).

C'est cette multiplicité à strictement parler incalculable des signes et surtout des emplois de chacun d'eux qui les rend à tout instant - «de seconde en seconde», pour reprendre une expression utilisée à propos du nom des Dieux - propres à subir déplacements et mutations. Rien de tel pour la légende, qui se transmet aussi dans le temps, mais dont les symboles, infiniment moins nombreux, donnent lieu à un nombre de mutations tout compte fait accessible au calcul.

51 J'ai conscience, au moment où je termine cet exposé, d'avoir peut-être excessivement donné dans la philologie saussurienne. J'y ai été contraint par la forme même du texte saussurien. La pensée de Saussure reste - et, par la force des choses restera définitivement - en gestation et en transformation. Peut-être est-elle, par ce trait même, l'image des problèmes qu'elle traite : ceux des relations entre linguistique et sémiologie.

\section{BIBLIOGRAPHIE}

Arrivé M. 1998 : « Unité linguistique et unité sémiologique chez Ferdinand de Saussure », in Quiroz G., Berthoud-Papandropoulo I, Thommen É. et Vogel C. (eds), Les unités discursives dans l'analyse sémiotique, Peter Lang, p. 11-21.

- 2000a, « Saussure, Barthes, Greimas », Modèles linguistiques, Tome XXI, fascicule 1, p. 19-37.

-2000 b : "Préface mêlée de souvenirs sur la préhistoire de la sémiotique », in Greimas A. J., La mode en 1830, P.U.F., p. XI-XXV.

Avalle S. d'Arco 1973 : «La sémiologie de la narrativité chez Saussure », in Bouazis Ch. (éd), Essais de la théorie du texte, Paris, éditions Galilée, p. 19-49.

CLG : voir Saussure 1916-1922-1985.

Cohen M. 1958 : La grande invention de l'écriture, Paris, Imprimerie Nationale et Klincksieck.

Engler R. 1974-1975 : « Sémiologies saussuriennes I », Cahiers Ferdinand de Saussure 29, p. 45-73.

- 1980 : « Sémiologies saussuriennes II », Cahiers Ferdinand de Saussure 34, p. 1-16. 
Fehr J. 2000 : Saussure entre linguistique et sémiologie, Paris, P.U.F.

Gandon F. 2002 : De dangereux édifices, Saussure lecteur de Lucrèce, à paraître.

Kim S. 1993 : «La mythologie saussurienne : une nouvelle vision sémiologique ? (À propos de la continuité de la pensée saussurienne) », Semiotica, 97, 1/2, p. 5-78.

Lacan J. 1966 : Écrits, Paris, Le Seuil.

LEG : voir Saussure 1986.

Naville A. 1901-1991 : Nouvelle classification des sciences, Paris, Félix Alcan, puis Didier Erudition.

Normand Cl. 2000 : Saussure, Paris, Les Belles Lettres.

Sandomir Dr I. L. LXXXVI È[re] P[ataphysique] : Opus pataphysicum, Testament de sa Feue

Magnificence le Docteur I. L. Sandomir, de son vivant Vice-Curateur Fondateur du Collège de 'Pataphysique, Collège de 'Pataphysique.

Saussure F. de 1916-1922-1985 [CLG] : Cours de linguistique générale, Lausanne-Paris, puis Paris, Payot.

- 1922-1984 : Recueil des publications scientifiques, Genève, Sonor et Lausanne, Payot, puis ParisGenève, Slatkine.

- 1986 [LEG] : Le leggende germaniche, scritti scelti e annotati a cura di Anna Marinetti e Marcello Meli, Este (Padova), libreria editrice Zielo.

- 1989 : Cours de linguistique générale, Édition critique par Rudolf Engler, tome 1, Wiesbaden, Harrassowitz.

- 1990 : Cours de linguistique générale, Édition critique par Rudolf Engler, tome 2, Wiesbaden, Harrassowitz.

\section{NOTES}

1. Saussure connaissait bien ce problème, notamment par les publications de son frère René, président de la Société espérantiste suisse, et auteur de nombreux travaux sur l'esperanto.

2. Pour un inventaire apparemment exhaustif de ces mentions de la sémiologie (et de la signologie, terme qui fut un moment utilisé par Saussure) voir Engler 1980.

3. Le sens a donner à avec n'est pas évident. Je pense pour ma part qu'il n'est pas instrumental (" en utilisant la langue »), mais comitatif (« en même temps que la langue »).

4. On a souvent constaté avec étonnement qu'en 1920, dans la $3^{\mathrm{e}}$ édition de son ouvrage - la Nouvelle classification avait été précédée, en 1888, d'une Classification des sciences - il n'est plus du tout question de la sémiologie. Certes, en 1920, le Cours est paru, et bien connu à Genève. Mais la sémiologie est encore, et pour de nombreuses années, en sommeil. C'est sans doute ce sommeil qui explique le silence de Naville.

5. On constate dans les sources manuscrites que Saussure n'a pas allégué l'exemple du mot Messieurs, mais celui du mot guerre (Saussure 1989 : I-244), puis de la succession alka-ok (I-414).

6. Cette "parenté » est explicitement alléguée, par exemple dans le passage suivant: "On s'aperçoit dans ce domaine, comme dans le domaine parent [souligné par M.A.] de la linguistique, que toutes les incongruités de la pensée proviennent d'une insuffisante réflexion sur ce qu'est l'identité » (LEG: 191).

7. Est-il utile de préciser que le mot symbole est utilisé avec le sens qu'a signe dans le CLG, comme le prouve son emploi pour désigner les « mots de la langue »?L'innovation consistant à réserver 
le terme symbole à cet objet oxymorique - et d'ailleurs impossible dans la langue - que serait le signe motivé est spécifique au CLG. En 1894, dans le projet d'article relatif à Whitney, Saussure utilise les termes symbole conventionnel et symbole indépendant avec le sens qui sera conféré dans le CLG à signe arbitraire (Saussure $1989: \mathrm{I}-23$ ).

8. On est tenté de penser à la 'pataphysique qui, selon l'aphorisme du Docteur Irénée Louis Sandomir, «se passe même d'être, car elle n'a même pas besoin d'être pour être " (Sandomir, LXXXVI, p. 151). Et l'on pense aussi à la dénégation lacanienne "il n'y a pas de métalangage » (Lacan 1966, passim), qui présuppose en se formulant l'être même qu'elle dénie. Visiblement, $l^{\prime}$ » être inexistant » a troublé beaucoup de commentateurs, à commencer par Avalle, Engler et Fehr.

9. Saussure fait allusion à cet emploi des runes, dans une optique qui évoque le problème des anagrammes, dont la pratique n'est pas absente de la recherche sur la légende (LEG 326).

10. Voir Cohen 1958 : 195-198. Toutefois les renseignements donnés par Saussure p. 30-31 de LEG sur la rune «appelée mystiquement zann »ne sont pas exactement confirmés par Cohen. Il faudrait rechercher les sources utilisées par Saussure pour ses remarques sur l'alphabet runique.

11. On a entrevu ce qu'ils sont pour le personnage légendaire comme pour la lettre. Pour le signe linguistique Saussure reste dans l'implicite. Engler (1974-1975 : 71) s'est essayé à expliciter, avec des résultats vraisemblables, sans plus : il parvient à une énumération de quatre éléments (signifié, signifiant, "parasémie» [statut fondé sur les associations paradigmatiques], syntagmatique). Le nombre est le même que celui qui est allégué pour la lettre (quatre) et très voisin de celui qui est affecté au personnage (de quatre à six selon les segments).

\section{RÉSUMÉS}

La sémiologie a été une des préoccupations les plus constantes de Saussure. Toutefois, on observe une étrange dissymétrie entre le statut qu'elle a dans la version standard du CLG et celui qui lui est réservé dans la recherche sur la légende. Saussure oscille entre deux points de vue opposés : tantôt le signe linguistique est donné comme un objet absolument spécifique, décourageant toute comparaison. Tantôt il est mis sur le même plan que d'autres objets sémiologiques, notamment le personnage de la légende. On cherche dans cet article à repérer les traits communs et les différences entre le signe linguistique et le personnage légendaire, respectivement qualifiés $d^{\prime}$ ' êtres nuls en soi » et d'« êtres inexistants ».

Saussure applied a constant attention to what be called semiology, but we can point out that his position on this matter was quite different whether he considered german legends or general linguistics. We shall try here to put forth common features as well as different ones between the linguistic sign and the legend's character.

\section{AUTEUR}

\section{MICHEL ARRIVÉ}

Professeur à l'Université de Paris X-Nanterre, CERES - CNRS FRE 2208, Michel.Arrive@wanadoo.fr 